and outpatient care services participated in our survey. Results: $78 \%$ of the facilities and mobile nursing services sustained migrants who accounted for app. $11.0 \%$ of the entire clientele. This value was considerably higher in urban areas such as Stuttgart $(15.8 \%)$ or Rhine-Neckar (15.4\%), when compared to rural areas such as the Black Forest (10.8\%). The proportion of migrants in care facilities corresponded to their proportion in the general population. There was a large heterogeneity regarding the countries of origin with migrants from Russia forming the largest group followed by citizens from Poland and citizens from Turkey. In contrast to the other migrant group the latter were underrepresented in inpatient but not outpatient care facilities. Practically all facilities employed migrants, especially as caregivers. Conclusions: Basically, citizens with a migration background seek help and support in both in and outpatient facilities just as citizens without this background do. This finding applies to both, urban and albeit to a lesser extent rural areas of Baden- Württemberg. It is suggested that the number of migrants in nursing homes will increase as their proportion in the population is higher among younger when compared to older birth cohorts.

\section{P1-254 INVESTIGATING THE SYNERGISTIC} RELATIONSHIP BETWEEN SLEEP QUALITY, PHYSICAL ACTIVITY, AND LEVELS OF BRAIN BETA-AMYLOID

Belinda M. Brown ${ }^{1}$, Stephanie R. Rainey-Smith ${ }^{2}$, Victor L. Villemagne ${ }^{3}$, Jeremiah J. Peiffer ${ }^{4}$, Sabine Bird ${ }^{1}$, Simon M. Laws ${ }^{5}$, Kevin Taddei ${ }^{5}$, Lance Macaulay ${ }^{6}$, Christopher C. Rowe ${ }^{7}$, David Ames ${ }^{8}$, Colin L. Masters ${ }^{3}$, Ralph N. Martins ${ }^{2},{ }^{1}$ School of Medical Sciences, Edith Cowan University, Perth, Australia; ${ }^{2}$ Sir James McCusker Alzheimer's Disease Research Unit (Hollywood Private Hospital), Perth, Australia; ${ }^{3}$ The Florey Institute of Neuroscience and Mental Health, Parkville, Australia; ${ }^{4}$ School of Psychology and Exercise Science, Perth, Australia; ${ }^{5}$ Edith Cowan University, Perth, Australia; ${ }^{6}$ CSIRO, Melbourne, Australia; ${ }^{7}$ Austin Health, Melbourne, Australia; ${ }^{8}$ National Ageing Research Institute, Melbourne, Australia. Contact e-mail: belinda.brown@ecu.edu.au

Background: There is currently no effective treatment for Alzheimer's disease (AD), thus, attention has turned to preventative measures, such as lifestyle modifications, that have the potential to delay AD onset. Recently, higher levels of physical activity and good sleep quality have been separately linked to lower levels of brain beta-amyloid $(\mathrm{A} \beta)$. In particular, our team has reported an association between higher levels of physical activity and lower brain $\mathrm{A} \beta$ in apolipoprotein $(A P O E) \varepsilon 4$ carriers. However, to our knowledge, no previous research has investigated whether a synergistic link between sleep and exercise exists, and the effect of such a link on AD-related neuroimaging biomarkers. Thus, we aim to investigate whether our previously reported association between higher levels of physical activity and lower brain $\mathrm{A} \beta$ in $A P O E \varepsilon 4$ carriers remains salient in individuals experiencing poor sleep quality. Methods: Data from 79 cognitively healthy men and women aged over 60 years was collected via the Australian Imaging, Biomarkers and Lifestyle (AIBL) study of Ageing. Subjective measures of physical activity and sleep quality were collected using the International Physical Activity Questionnaire and Pittsburgh Sleep Quality Index (PSQI), respectively. Based on answers given to the PSQI, participants were classified as having either 'good' or 'poor' sleep quality. Brain $\mathrm{A} \beta$ burden was quantified by amyloid-binding positron emission tomography ligands. The association between sleep, physical activity and brain
$\mathrm{A} \beta$ was evaluated using multiple linear regression analyses. Results: The association between higher levels of physical activity and lower brain $\mathrm{A} \beta$ in $A P O E \varepsilon 4$ carriers was evident in participants reporting good sleep quality $(\mathrm{F}=3.69, p<0.05)$; this result was not salient in those reporting poor sleep quality $(\mathrm{F}=0.33, p>0.10)$. Further analyses will increase the sample size, and also evaluate other factors (i.e. other genotypes) as potential moderators of the link between sleep, physical activity and $\mathrm{A} \beta$. Conclusions: Our results suggest that a combination of physical activity and good sleep quality may contribute to maintaining cognitive health, and through interpretation of $\mathrm{AD}$-related neuroimaging results, may indicate a protective effect on the ageing brain, leading to a reduced risk or delay in onset of $\mathrm{AD}$.

\section{P1-255 LIFESTYLE, TREATMENT, AND COGNITIVE DECLINE IN ELDERLY DIABETICS}

Ilianna Lourida ${ }^{1}$, Elzbieta Kuzma ${ }^{1}$, William E. Henley ${ }^{1}$, Jo ThompsonCoon $^{1}$, Chris M. Dickens ${ }^{1}$, Kenneth M. Langa ${ }^{2}$, David J. Llewellyn ${ }^{1}$, ${ }^{1}$ University of Exeter, Exeter, United Kingdom; ${ }^{2}$ University of Michigan, Ann Arbor, MI, USA. Contact e-mail: i.lourida@exeter.ac.uk

Background: Diabetes is associated with accelerated cognitive decline and risk of dementia. No prospective study has examined the associations between lifestyle and treatment factors included in diabetes management and cognitive function in older adults with diabetes, and whether these associations are mediated by glycaemic control. Methods: We used data from the 2003 Health and Retirement Study (HRS) Mail Survey on Diabetes and five waves of the HRS between 2002 and 2010 with follow-up cognitive assessments every two years. Change in cognitive function was assessed using a modified version of the Telephone Interview for Cognitive Status. Lifestyle and treatment factors were assessed by self-report. $\mathrm{HbA}_{1 \mathrm{c}}$ levels were measured to allow the investigation of glycaemic control as a potential mediator. Missing data were imputed using multiple imputations with chained equations. Multilevel mixed-effects models were used to determine the association of i) diet, ii) alcohol consumption, iii) physical activity, iv) diabetes treatment regimen, and v) treatment adherence with cognitive decline adjusted for potential confounders. Results: A total of 931 adults with diagnosed diabetes aged 65 years and older (mean age 73 ( \pm 6.15$)$ years) at first cognitive assessment were included in the analysis. Following a healthy diet plan was associated with worse cognitive performance at baseline $(\beta=-0.16,95 \% \mathrm{CI}$ : -0.32 to $-0.002, p=0.047)$, and there was a trend of lower cognitive scores for those receiving a combination of oral medication and insulin compared to non-medically treated diabetics $(\beta=-1.10,95 \%$ CI: -2.24 to $0.03, p=0.057$ ). However, we did not observe significant associations between lifestyle, diabetes treatment and cognitive decline over $6.6( \pm 2.3)$ years of follow-up after adjustment for age, sex, race/ethnicity, education, depressive symptoms, smoking, diabetes duration and other diabetes management behaviours. The absence of significant associations precluded the assessment of glycaemic control as a potential mediator. Conclusions: In this relatively healthy sample of elderly diabetics, lifestyle and diabetes treatment were not associated with cognitive decline during follow-up. Additional studies in more heterogeneous populations with undiagnosed and known diabetes are needed to determine whether lifestyle modifications and treatment options encountered in diabetes management are related to cognitive decline in the elderly. 